\title{
Which Factor Determines the Duration Required for Relief of Glucotoxicity by the Intensive Insulin Therapy?
}

\author{
Hidekatsu Yanai $^{\mathrm{a}, \mathrm{b}}$, Mariko Hakoshima ${ }^{\mathrm{a}}$, Hiroki Adachi ${ }^{\mathrm{a}}$
}

\section{To the Editor}

Hyperglycemia exerts deleterious effects on pancreatic $\beta$-cell function, which is called as glucotoxicity [1]. Several mechanisms including high glucose-induced $\beta$-cell overstimulation, oxidative stress and protein glycation may contribute to the development of glucotoxicity [1]. It was suggested that the beneficial effect of early transient intensive insulin therapy at diagnosis rapidly limits the damage caused by glucotoxicity, improving residual $\beta$-cell function and potentially slowing disease progression [2].

In National Center for Global Health and Medicine Kohnodai Hospital, Japan, an early transient intensive insulin therapy is actively performed in patients who are hospitalized due to hyperglycemia. Diabetes specialists roughly adjust insulin dose to achieve blood glucose levels of less than $130 \mathrm{mg} /$ $\mathrm{dL}$ before every meal and at bedtime. When the daily required dose of insulin began to decrease, it is clinically considered that glucotoxicity has been relieved. After that, we measure serum fasting and postprandial C-peptide levels and daily urinary C-peptide level to evaluate intrinsic insulin secretary capacity. We determine the eventual treatment for diabetic patients by referring intrinsic insulin secretary capacity.

To elucidate which factor determines the duration required for relief of glucotoxicity by the intensive insulin therapy, we retrospectively studied patients hospitalized for treatment of type 2 diabetes, who had been treated by the intensive insulin therapy between June 2014 and May 2017. This study was approved by the Institutional Ethics Committee in National Center for Global Health and Medicine, and was also performed in accordance with the Declaration of Helsinki.

We found 53 eligible patients (male/female, 27/26). The mean \pm SD of age, body mass index (BMI) and hemoglobin A1c $(\mathrm{HbA} 1 \mathrm{c})$ were $65.3 \pm 17.4$ years old, $26.6 \pm 6.9 \mathrm{~kg} / \mathrm{m}^{2}$, and $11.4 \pm 2.2 \%$, respectively. We picked up data including serum li-

Manuscript submitted March 28, 2018, accepted April 13, 2018

a Department of Internal Medicine, National Center for Global Health and Medicine Kohnodai Hospital, Chiba, Japan

${ }^{\mathrm{b}}$ Corresponding Author: Hidekatsu Yanai, Department of Internal Medicine, National Center for Global Health and Medicine Kohnodai Hospital, 1-7-1 Kohnodai, Ichikawa, Chiba 272-8516, Japan.

Email: dyanai@hospk.ncgm.go.jp

doi: https://doi.org/10.14740/jocmr3423w pids, liver and renal function, area under the curve (AUC) of blood glucose, serum and urinary C-peptide levels and insulin dose.

To obtain the relief of glucotoxicity by the intensive insulin therapy, $8.9 \pm 4.3$ days were required. The AUC of blood glucose at the start of intensive insulin therapy significantly decreased from $5,282 \pm 1,406$ to $3,670 \pm 822 \mathrm{mg} / \mathrm{dL}(\mathrm{P}<0.0001$ by paired $t$-test $)$ after the relief of glucotoxicity. Daily insulin dose significantly increased from $17.7 \pm 7.8$ to $25.0 \pm 14.9$ units $(\mathrm{P}<0.0001)$.

The duration required to relieve glucotoxicity was not correlated with age, BMI, HbA1c, liver and renal function, serum lipid levels, and serum fasting and postprandial C-peptide levels and daily urinary C-peptide level. The duration required to relieve glucotoxicity was positively and significantly correlated with daily insulin dose when glucotoxicity had been relieved (Fig. 1a), and the AUC of blood glucose at the start of intensive insulin therapy (Fig. 1b). Furthermore, the duration required to relieve glucotoxicity was negatively and significantly correlated with the duration between the start of intensive insulin therapy and the admission $(\mathrm{r}=-0.404, \mathrm{P}=0.003)$. Daily insulin dose used when glucotoxicity had been relieved was positively and significantly correlated with BMI (Fig. 2a), HbAlc (Fig. 2b), serum triglyceride (Fig. 2c) levels at the start of intensive insulin therapy.

Our study suggested that it takes more duration to relieve glucotoxicity by the intensive insulin therapy in patients with greater AUC of blood glucose at the start of insulin therapy who needed more daily insulin dose. An early start of insulin therapy after the admission may shorten the duration required to relieve glucotoxicity. Further, our study showed that more daily insulin dose may be required in patients with poorer glycemic control, higher levels of BMI and serum triglyceride, which supports the significant influences of insulin resistance and lipotoxicity on glucotoxicity [3].

The present study has several limitations. First, other hypoglycemic, anti-hypertensive, or lipid lowering agents, food intakes and/or exercise levels may have an influence on the study results. Second, the number of studied subjects was small because of the limited availability. However, the present study also has the strength. First, metabolic parameters were measured by the same laboratory. Second, subjects were treated by the specialists for diabetes treatment who were equally educated by the same specialist.

In conclusion, our study elucidated the factors which influence on duration and daily insulin dose to relieve glucotoxicity by the intensive insulin therapy among Japanese patients with type 2 diabetes hospitalized due to hyperglycemia. 


\section{$\mathbf{a}$}
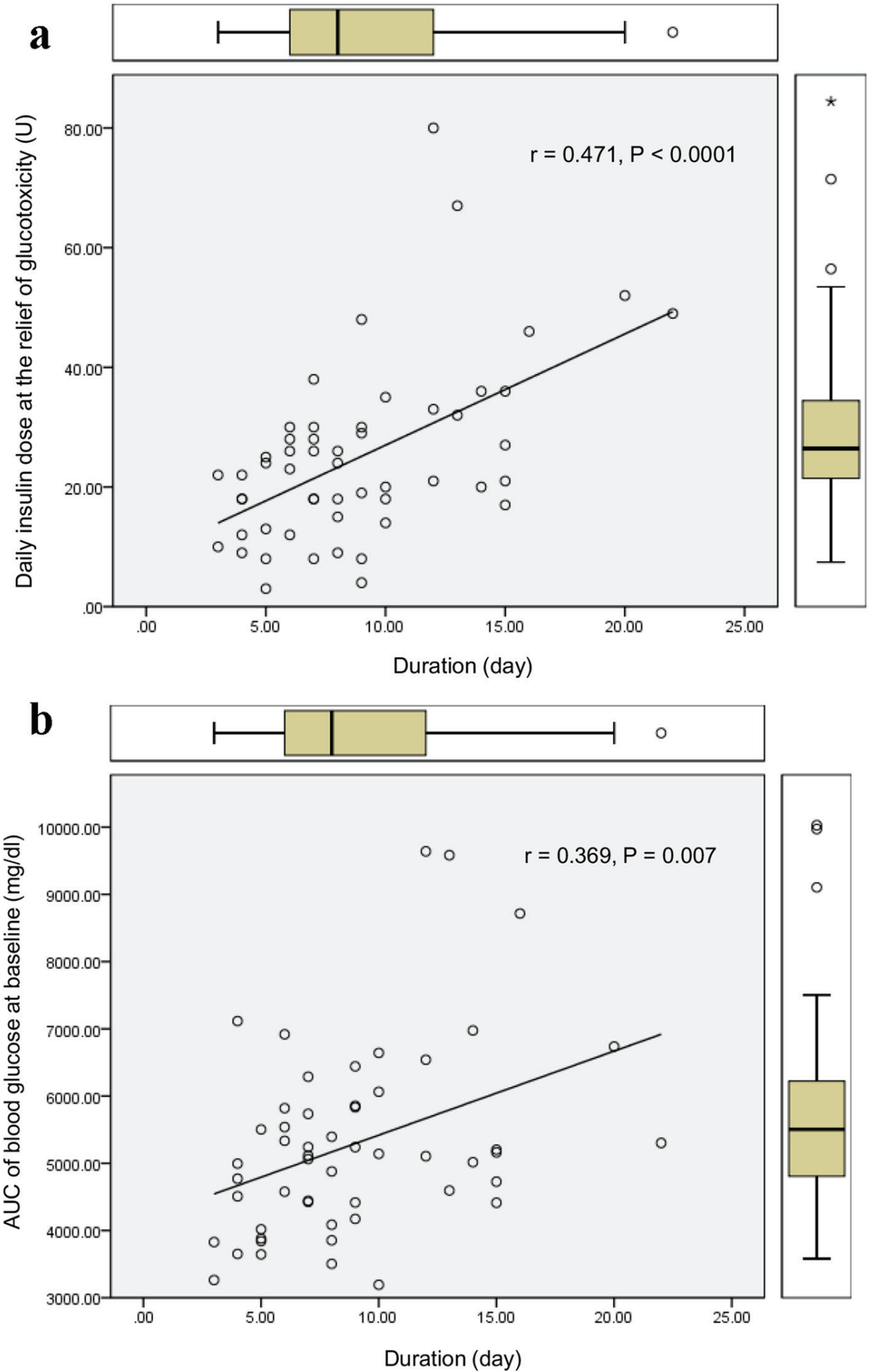

Figure 1. Correlations of the duration required to relieve glucotoxicity with daily insulin dose when glucotoxicity had been relieved (a), and the AUC of blood glucose at the start of intensive insulin therapy (b). $r$ indicates correlation coefficient, and a statistical analysis was performed by the Spearman's correlation. 

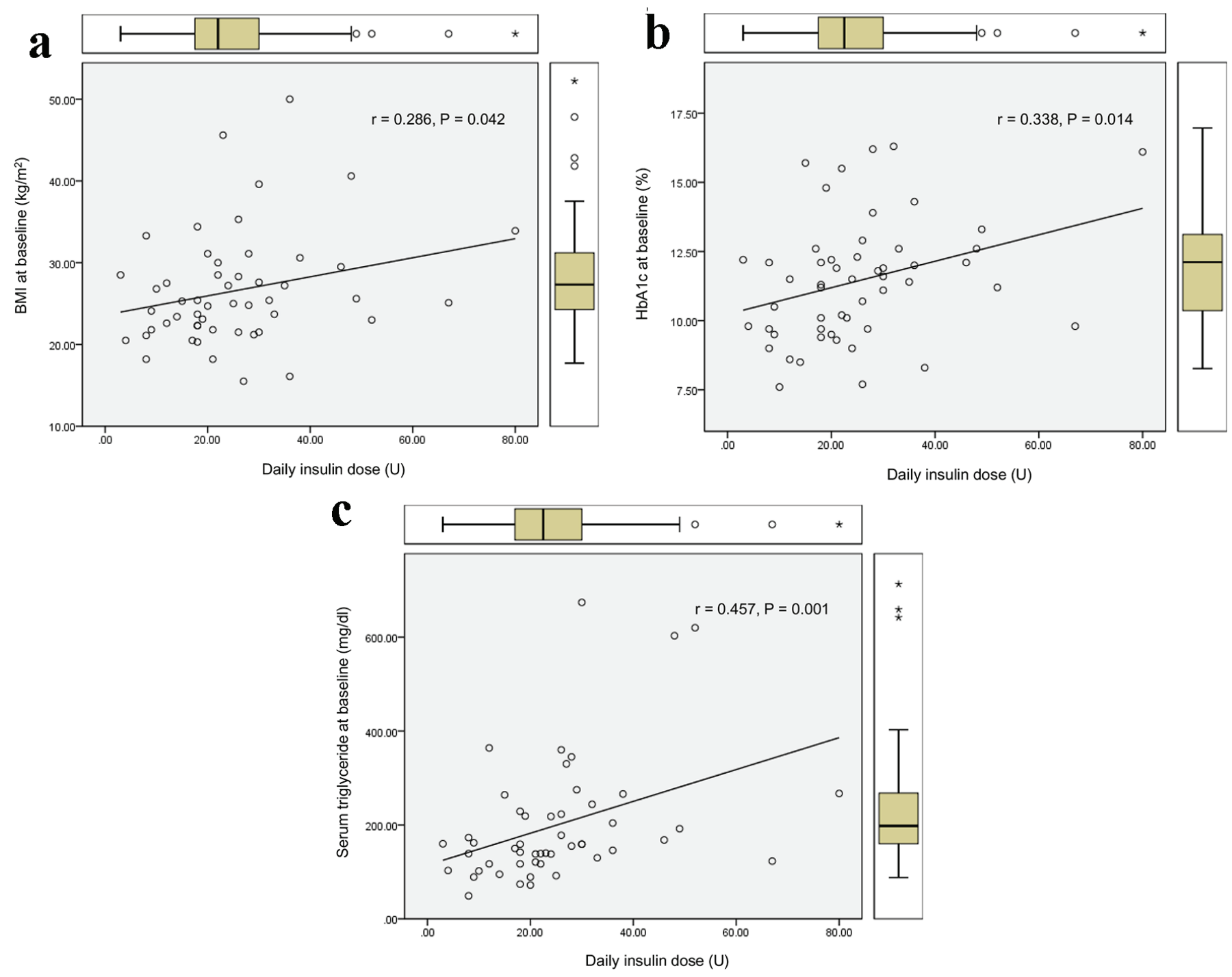

Figure 2. Correlations of daily insulin dose used when glucotoxicity had been relieved with BMI (a), HbA1c (b), and serum triglyceride (c) levels at the start of intensive insulin therapy. $r$ indicates correlation coefficient, and a statistical analysis was performed by the Spearman's correlation.

\section{Conflict of Interest}

The authors declare that they have no conflict of interest concerning this article.

\section{References}

1. Bensellam M, Laybutt DR, Jonas JC. The molecular mechanisms of pancreatic beta-cell glucotoxicity: recent findings and future research directions. Mol Cell Endocrinol. 2012;364(1-2):1-27.

2. Hanefeld M. Use of insulin in type 2 diabetes: what we learned from recent clinical trials on the benefits of early insulin initiation. Diabetes Metab. 2014;40(6):391-399.

3. Seghieri M, Trico D, Natali A. The impact of triglycerides on glucose tolerance: Lipotoxicity revisited. Diabetes Metab. 2017;43(4):314-322. 